\title{
Methodology Report
}

\section{An Experimental Model for Resistance Exercise in Rodents}

\author{
Humberto Nicastro, ${ }^{1}$ Nelo Eidy Zanchi, ${ }^{2}$ Claudia Ribeiro da Luz, ${ }^{1}$ \\ Daniela Fojo Seixas Chaves, ${ }^{1}$ and Antonio Herbert Lancha Jr. ${ }^{1}$ \\ ${ }^{1}$ Laboratory of Applied Nutrition and Metabolism, School of Physical Education and Sports, University of São Paulo, \\ Cidade Universitária, 05508-030 São Paulo, SP, Brazil \\ ${ }^{2}$ Department of Physiology and Biophysics, Institute of Biomedical Sciences, University of São Paulo, 05508-030 São Paulo, SP, Brazil
}

Correspondence should be addressed to Humberto Nicastro, nicastro@usp.br

Received 6 August 2011; Revised 21 October 2011; Accepted 28 November 2011

Academic Editor: Leon Spicer

Copyright ( $\odot 2012$ Humberto Nicastro et al. This is an open access article distributed under the Creative Commons Attribution License, which permits unrestricted use, distribution, and reproduction in any medium, provided the original work is properly cited.

\begin{abstract}
This study aimed to develop an equipment and system of resistance exercise (RE), based on squat-type exercise for rodents, with control of training variables. We developed an operant conditioning system composed of sound, light and feeding devices that allowed optimized RE performance by the animal. With this system, it is not necessary to impose fasting or electric shock for the animal to perform the task proposed (muscle contraction). Furthermore, it is possible to perform muscle function tests in vivo within the context of the exercise proposed and control variables such as intensity, volume (sets and repetitions), and exercise session length, rest interval between sets and repetitions, and concentric strength. Based on the experiments conducted, we demonstrated that the model proposed is able to perform more specific control of other RE variables, especially rest interval between sets and repetitions, and encourages the animal to exercise through short-term energy restriction and "disturbing" stimulus that do not promote alterations in body weight. Therefore, despite experimental limitations, we believe that this RE apparatus is closer to the physiological context observed in humans.
\end{abstract}

\section{Introduction}

Muscle strength and power have been considered strong exogenous stimuli that are able to improve sports performance and promote therapeutic effects in several pathological conditions characterized by muscle wasting, such as cancer, disuse, sepsis, and sarcopenia $[1,2]$. In this context, resistance exercise (RE) has demonstrated significant effects regarding neural [3], metabolic [4], and functional adaptations [5] in skeletal muscle. For this reason, recent studies in humans and rodents have focused on the mechanisms behind such responses promoted by RE, especially molecular and phenotypic characterization [6-9]. Although human studies provide strong evidence for practical application (i.e., greater external validity), it is not always possible to control the variables that may influence a biological response. Furthermore, collection of muscle tissue samples in order to provide a significant body of cellular evidence $[10,11]$ may limit mechanistic human studies. Therefore, experimental research has been used to provide primary results that may support subsequent studies in humans.
Since the study of Klitgaard [12], literature has described some experimental models of $\mathrm{RE}$ for rodents that have presented interesting results concerning skeletal muscle adaptations [13-16]. These studies have demonstrated that rodents are able to perform long- and short-term RE with different RE protocols and that their hindlimb muscle adapts similar to human muscles in terms of size, fiber type profile, and functionality. Furthermore, molecular characterization of RE effects have provided some insight in terms of cell signaling pathways (transcription and translation) involved in the control of skeletal muscle growth (hypertrophy, atrophy, and inflammation) and functionality (strength capacity).

However, these experimental models present limitations that may compromise the extrapolation of data to humans. There are many factors that can limit experimental models of $\mathrm{RE}$ in comparison to those performed in humans and, among these, we can cite as the main one, the stimulus that is needed to encourage the animal to execute the exercise. Indeed, environmental stress is required, but many models use fasting and electric shock as reward and punishment 
stimulus, respectively, which is beyond the physiological context observed in humans, and could be considered a limiting factor in experimental models characterized by appetite suppression and energy restriction. Some models also report that animals are not allowed to be "free" in a given area and remain "tied" in the training apparatus during exercise session. Regarding the exercise physiology context, these experimental models do not provide appropriate control of RE variables: volume (series and repetitions), intensity, and rest interval between repetitions and sets. This lead us to question whether the stimuli is quantitatively enough or not for muscle tissue in terms of volume and overload.

Thus, the aim of the present report is to propose an experimental model of RE for rodents that could mimic (considering the limitation of the species) the training carried out in humans. We developed an RE model that does not use fasting nor electric shock as stimuli for exercise execution, allows operant conditioning so that the animal does not stay "tied" to the training apparatus, and promotes the control of RE variables to perform specific muscle function tests. With this model it is possible to minimize environmental stress, to approach the animal in a physiological context, to study skeletal muscle adaptations in response to RE in several experimental conditions, and to compare the responsiveness of rodents' and humans' muscles to different RE protocols.

\section{Material and Methods}

2.1. Animals. The experiments were conducted in accordance with the National Research Council's Guidelines for the Care and Use of Laboratory Animals. This study was approved by the Local Ethical Committee. Six Wistar male rats (400-450 g) were housed under controlled environmental conditions (temperature, $22^{\circ} \mathrm{C}$; 12 -h dark period starting at $18: 00 \mathrm{~h})$. They were given free access to commercial laboratory chow and water before the experiments were performed. During periods of energy restriction, food intake was reduced to $25 \%$ of basal intake.

2.2. Apparatus Description. The whole equipment consists of an RE apparatus and an interface of control (hardware) on the rear panel of the equipment and was based on squattype studies of Klitgaard [12] and Wirth et al. [15]. Figures 1 and 2 illustrate the training apparatus and its control devices. The RE apparatus has its external structure built in acrylic, preferably dark, which holds all devices of the equipment and keeps the main tool of the experiment: the weight lifting cylinder. Thus, its composition can be divided to the acrylic structure, rear panel, drawer, copper bars, cylinder lid, weight lifting cylinder, sensors, and actuators.

(i) Acrylic structure (Figure 1, Item 1): the acrylic structure is bicompartmentalized, that is, it has two divisions: the lower box, to house devices of communication, sensors, actuators, and electronic boards, and the higher box, to place the animal and the weight lifting cylinder. Each compartment has distinct lids which have a slot for passage of sensor wires.

(ii) Rear panel (Figure 1, Item 2): in the rear panel the connectors responsible for power supply and the hardware are allocated.

(iii) Drawer (Figure 1, Item 8; Figure 2, Item 9): it is an aluminum tray that lines the bottom of the higher box during the experiments, facilitating the removal of animal wasting. It can be washed with water and is usually covered with paper for easy cleaning.

(iv) Copper bars (Figure 1, Item 7; Figure 2, Item 8): responsible for supporting the weight lifting cylinder and can be exchanged for another metal material.

(v) Cylinder lid: this device is attached on the top of the weight lifting cylinder to provide better fixation of the cylinder to the acrylic structure, avoiding fluctuations during the experiment due to the rise and fall of weights.

The weight lifting cylinder is located inside the RE apparatus and is composed by the following.

(i) Acrylic cylinder (Figure 2, Item 1): it is a holder that allows the animal to lift the weight as squat-type exercise. In this cylinder are coupled: weight plates, lifting ring, lifting bar, and the measurement axis.

(ii) Lifting ring (Figure 2, Item 6): it is an acrylic piece which is located inside the acrylic cylinder, with one side attached to the measurement axis and the other to a vertical "rail" found in the body of the acrylic cylinder. The height of this device can be adjusted according to the size and species of the animal.

(iii) Lifting bar (Figure 2, Item 10): it is a steel piece responsible for supporting weight plates for the lifting movement. Weight plates must be placed at the predetermined distances relative to the "rail" found in the acrylic cylinder.

(iv) Weight plates: $50 \mathrm{~g}$ cylinders that should be placed in the lifting bar and fixed with the aid of elastics (i.e., orthodontic elastics). More than one weight plate can be placed in the lifting bar at a time, but they should stay together side by side. The point at which they are placed on the lifting bar must be considered as the midpoint of the set of weight plates on "rail" of the acrylic cylinder.

(v) Axis measurement (Figure 2, Item 5): it consists of a potentiometer and an acrylic base. This axis should enable the establishment of the lifting ring trough a cross-bolt to the measurement axis. It enables the measurement of angular displacement of the lifting bar through the angle sensor. The wire must be protected from animal bites.

(vi) Support base (Figure 2, Item 7): it is responsible for acrylic cylinder fixation to the copper bars. The support base consists of two symmetrical parts and must be put side by side below the acrylic cylinder. 


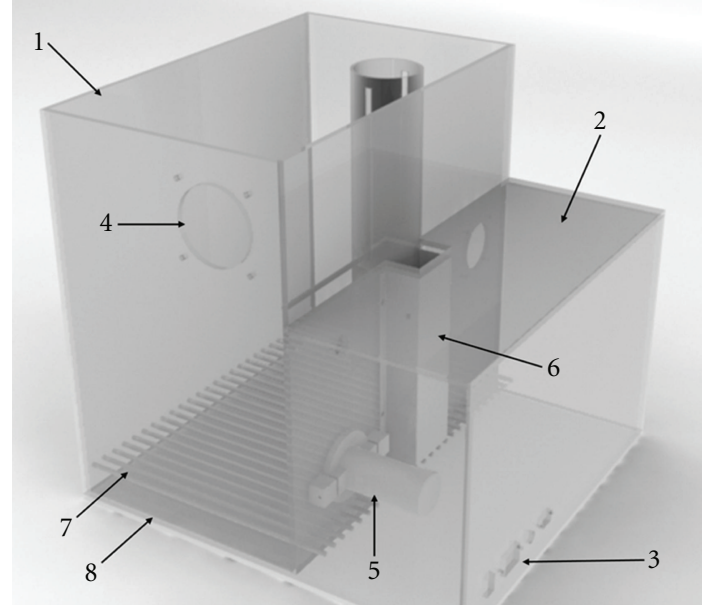

Figure 1: External view of the equipment. 1: acrylic structure; 2: rear panel; 3: power and interface plugins; 4: fan; 5: nose poke; 6: automatic feeder; 7: copper bars; 8: drawer.

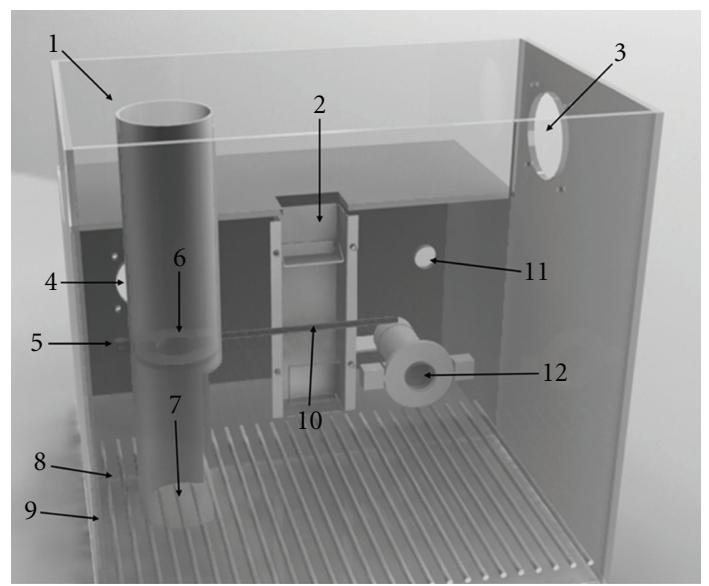

FIGURE 2: Internal view of the equipment. 1: weight lifting cylinder; 2: automatic feeder; 3 : fan; 4: sound device; 5 : axis measurement; 6 : lifting ring; 7: support base; 8: copper bars; 9: drawer; 10: lifting bar; 11: light device; 12: nose poke.

The set is fixed with a screw the "U" shape on both sides of the support base allows its attachment to the copper bars.

The sensors are responsible for collecting specific information about the experiment. The sensors are as follows.

(i) Angle sensor: it is the sensor responsible for measuring the angle of the lifting bar. The angle sensor is attached to the weight lifting cylinder acting as the axis of the rotation of the lifting bar, allowing the software to calculate the angular displacement of the lifting bar when it is rotated. From this and other data, the software can also calculate the concentric and eccentric strength exerted by the animal as well as the length of each movement.

(ii) Nose poke (Figure 1, Item 5; Figure 2, Item 12): it is through this device that the system receives each "nose poking" signal of the animal. This device is used for conditioning the animal to the training protocol. It has a circular hole in which the animal introduces the nose cutting an infrared light and triggering the device. At the bottom of the hole there is as light-emitting diode (LED) that emits yellow light, in order to stimulate the animal when it is operating.

The actuators, located inside the apparatus, are responsible for performing specific actions on the RE apparatus. The actuators are as follows.

(i) Automatic feeder (Figure 1, Item 6; Figure 2, Item 2): it is installed inside the apparatus. A pellet at a time is released, requiring replacement of a new pellet into the feeder after the previous release. The release of pellets occurs through a door controlled by a motor servo which is controlled by the hardware in the rear panel of the equipment. The site for pellets storage allows placing pellets of varying sizes.

(ii) Light device (Figure 2, Item 11): it is placed inside the apparatus in order to illuminate most of the distribution of acrylic structure through a lamp, preferably red and with low power. During the RE session this lamp can remain on or off.

(iii) Sound device (Figure 2, Item 4): it is placed inside the apparatus and generates noise through a speaker that is also located inside the equipment and protected by a grid. During the exercise session, the sound is activated as a conditioning tool (encourage/disturb the animal).

(iv) Fan (Figure 2, Item 3): it is installed into the upper compartment of the equipment and remains ventilating while the apparatus is on trial. It keeps the air circulation inside the equipment, produces a very low noise, and is not within the reach of the animal.

The present equipment has all the sensors and devices described highly integrated into its structure and is highly resistant, supporting all actions and movements of the animal. Moreover, it is not necessary to perform the assembly of each component individually prior to use and it is easy to operate and to clean.

2.3. Conditioning the Animal to RE. The phases of operant conditioning adopted in the present equipment are the same described by Wirth et al. [15]. However, we modified the logistics of such phases in order to optimize the exercise execution by the animal. As previously mentioned, we did not use fasting as a reward stimulus to execute the exercise, but for conditioning the animal. Importantly, environmental conditions are critical in the experiment. Animal conditioning is totally dependent of the variables light, sound, and smell. Therefore, the environment where the experiment was conducted was extremely well-controlled: dark (illuminated by a low-power red light), silent, and devoid of atypical odors. Briefly, the phases of operant conditioning were separated by $48 \mathrm{~h}$ and carried out as follows. 
(i) Magazine: in this phase we only placed the animal inside the apparatus to adapt to the environment. After $12 \mathrm{~h}$ of energy restriction the animal remained inside the equipment for 1 hour. During this period, pellets of $\sim 30-40 \mathrm{mg}$ were released (a total of 30 pellets) at regular intervals for the animal to identify the automatic feeder. It was necessary to supply the automatic feeder in each cycle. This procedure was performed only once.

(ii) Nose-poke 1: as shown in Figure 2, nose-poke is installed besides the automatic feeder. Again, the animal was placed inside the equipment after $12 \mathrm{~h}$ of energy restriction. The automatic feeder was triggered each time the animal introduced its nose into the nose-poke. This event was called "poking." A total of 30 cycles was established for each experiment. Based on our experience with the apparatus, some food smell in the nose-poke was necessary to encourage the animal to introduce its nose inside it. This phase was also performed only once.

(iii) Nose-poke 2: in the second phase of nose-poke, the sound device was integrated to the conditioning process. A constant noise was emitted and when the animal introduced its nose into the nose-poke, the automatic feeder was triggered and the noise stopped. The sound device was used to disturb the animal so that when the task ("poking") was performed correctly, the animal was food-rewarded and the noise stopped disturbing. This phase introduced a new variable in order to replace the food reward as the only way to encourage the animal to perform the task proposed. A total of 30 interleaved cycles of pellets (15 with and without food) release were performed. This phase was performed twice.

(iv) Standing 1: the nose-poke device was removed from the wall and placed inside the weight lifting cylinder, above the lifting ring, and a lid was placed in the nose-poke hole on the wall. The adaptation followed the same procedures of the previous phase. This phase was conducted to encourage the animal to enter into the weight lifting cylinder, but without lifting the ring, and was performed twice.

(v) Standing 2: in the second phase of standing, food reward was completely removed and the animal had the constant sound as the only stimulus to perform the task. Two sessions were required.

(vi) Lifting: in the last phase, the nose-poke device remained within the weight lifting cylinder, however, at an unreachable height to the animal. Using the software, a minimum lifting height was set depending on the species and size of the animal. Collar height can be adjusted according to the size of the animal. We observed that a height of $4 \mathrm{~cm}$ was necessary for an animal of $\sim 400 \mathrm{~g}$ to complete the movement (full concentric contraction). During the lifting task, animals performed concentric muscle contractions being plantaris and soleus the main muscles involved in contraction. Of note, the access of the lifting ring allowed the animal to pass only the head through the ring hole and its height was adjusted according to the animal's characteristics. The sound was turned on and once the animal raised the lifting bar to the pre-determined height, the noise stopped. It was necessary to carry out one session without load and one session with light load.

It is important to emphasize that the animal did not stay "tied" in the training apparatus. Once conditioned, the animal underwent a muscle function test: maximal voluntary strength capacity (MVSC) as previously described by our group [17] using the same apparatus and procedures described by Klitgaard [12]. The advantages of the present equipment are that the rest intervals between each repetition in the MVSC were optimized because of the sound stimulus, it was possible to calculate concentric and eccentric strengths of the animal during each muscular contraction and relativize the training intensity with better precision (percent of MVSC, percent of body weight). Additionally, the MVSC test can be performed during and at the end of the experimental protocol to assess the progression of muscle function.

To configure the training protocol in the software it was necessary to enter the following data: training volume (sets and repetitions), rest intervals between sets and repetitions, desired height of the lifting bar, and weight plate position in the lifting bar. The experiment could be interrupted at any time and all data were stored in a report file by the software.

2.4. Statistical Analysis. Data are expressed as mean \pm SEM. Differences between nose-poke-phases and body weight values were tested by paired $t$-test. The level of significance was set at $P<0.05$. Statistical analysis was performed using SAS 8.2 (SAS Institute Inc., Cary, NC, USA).

\section{Results}

As previously described, to condition the animal to the experimental model it was necessary to carry out periods of energy restriction on the previous day of nose-poke 1 and 2 and standing 1 phases. However, as shown in Figure 3, energy restriction did not promote significant changes in body weight at the end of the conditioning process (414.1 \pm $4.8 \mathrm{~g}$ in Pre versus $410.8 \pm 4.2 \mathrm{~g}$ in Post period; $P>0.05)$.

Figure 4 presents the time spent to complete each adaptation phase. Magazine phase is not described because it was carried out only to adapt the animal to the exercise environment. To complete the 30 cycles proposed in the nose-poke 1 phase, animals took $1868 \pm 289$ seconds. Interestingly, when the sound device was integrated to the conditioning (nose-poke 2 phase), animals took $58.9 \%$ less time to complete the cycles $(768.4 \pm 109.1$ seconds; $P=$ $0.03)$. It is important to emphasize that, although the animals were more conditioned to the "poking" task in nose-poke 2 phase, this factor did not contribute to reduce the time spent to complete the cycles. We repeated the nose-poke 1 phase (without sound) and observed reduction of time (data not shown). Standing phases time were not statistically 


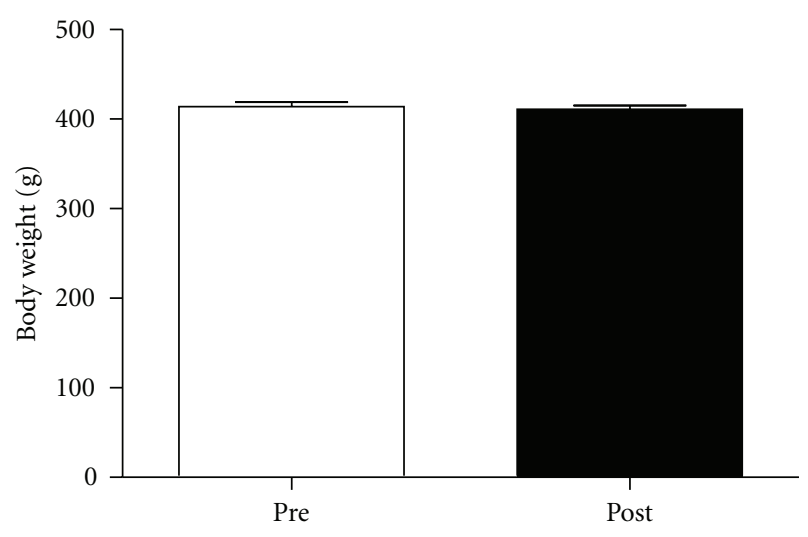

Figure 3: Body weight before (Pre) and at the end (Post) of the adaptation phases. Not significantly different $(P>0.05)$.

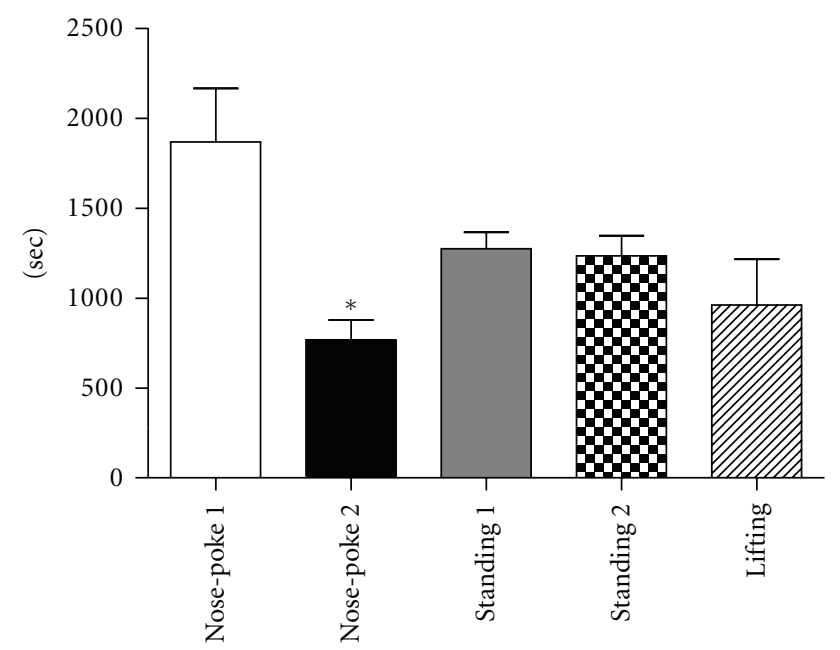

FIgUre 4: Time spent at each operant conditioning phase. Data are expressed as mean \pm SEM. ${ }^{*} P<0.05$ versus nose-poke 1 .

different $(1274 \pm 92.4$ seconds in standing 1 versus $1234 \pm$ 113.3 seconds in standing 2 phases; $P>0.05$ ). Animals took $960.8 \pm 254.2$ seconds to complete the lifting phases and lifted the bar to $4.1 \pm 0.1 \mathrm{~cm}$.

After the conditioning phases, animals performed the MVSC test. Height displacements during repetitions were quite similar to lifting phase and the result of the MVSC during the concentric phase of muscle contraction was $2.3 \pm$ $0.1 \mathrm{~N}$ (Figure 5). We did not describe the eccentric strength performed because this experimental model is totally based on concentric muscle actions, since during the eccentric phase the animal does not counteract the external load. The rest interval between each repetition to perform a repetition (contraction) was $7.2 \pm 0.8$ seconds (Figure 5). Thus, each time the sound stimulus was released, animals took approximately 7 seconds to perform the next repetition.

\section{Discussion}

The aim of the present report was to propose an experimental model of RE for rodents with better conditioning (no fasting

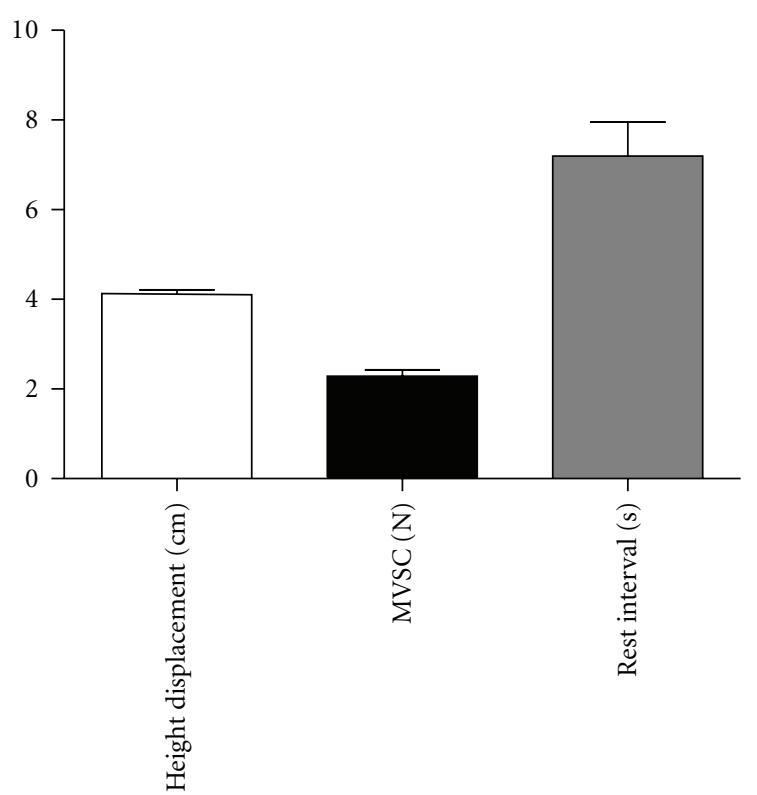

Figure 5: Height displacement $(\mathrm{cm})$, maximal voluntary strength capacity (MVSC) during the concentric phase of muscle contraction $(\mathrm{N})$, and rest interval between repetitions (s) during the functional test. The height displacement in MVSC test was the same observed in the lifting phase. Data are expressed as mean $\pm \mathrm{SEM} ; \mathrm{N}$ newton.

and shocking) and control of exercise variables (volume, intensity and rest intervals). The first topic to be discussed is the functionality of the apparatus. As previously described, animals were able to perform the conditioning phases within the logistics proposed. Second, integrating the sound device into the conditioning process optimized the performance of the exercise learning. Regarding exercise physiology, it was possible to optimize the encouragement of the animal to perform muscle contractions controlling repetitions and rest interval between repetitions. Therefore, it was possible to control and optimize the variable of RE "rest interval."

Importantly, although this experimental model uses "energy restriction" as the first stimulus to condition the animal to the exercise, it did not promote significant changes in body weight and, consequently, in muscle mass. This can be explained by the fact that the energy restriction was imposed only at the beginning and gradually abolished during the conditioning process. The applicability of such factor is quite important since models of skeletal muscle atrophy (i.e., glucocorticoids, fasting, cancer, and sepsis) are usually characterized by reduction in food intake. Thus, food reward would not be a strong stimulus. Furthermore, this experimental model demonstrates that it is not necessary to punish the animal (shocking) to encourage exercising. In this context, sound device proved to be a good "disturbing" variable that stimulates the animal to complete the task.

Recently, we conducted an experiment to evaluate the effectiveness of this experimental model in counteracting dexamethasone-induced skeletal muscle atrophy, an experimental model characterized by severe muscle wasting and appetite suppression. Even with loss of appetite, animals 
performed, during 7 days, 3 sessions of RE composed by 3 sets of 10 repetitions separated by 48 hours. We demonstrated that, although RE did not attenuate the atrophic response, it promoted significant phenotypic adaptations in plantaris muscles that in the long term may reflect structural remodeling. Furthermore, RE-trained animals presented higher concentric MVSC when compared with nontrained animals at the end of the experimental protocol. Therefore, the experimental mode is effective in conditioning the animal to RE and, according to the exercise protocol, to promote phenotypic, structural, and functional responses in skeletal muscle at least in dexamethasone-treated animals [18].

Although the present equipment promotes good control of RE variables, the main limitation concerns the strength data. Concentric and eccentric measurements are calculated by estimative, that is, not directly measured by a strength platform as proposed by Wirth et al. [15]. However, a strength platform can be easily incorporated into the equipment. Furthermore, it proposes a more specific control of other RE variables, especially rest interval between sets and repetitions. Furthermore, it encourages the animal to exercise through short-term energy restriction and "disturbing" stimuli that do not promote alterations in body weight. Therefore, we believe that this RE apparatus is closer to the physiological context observed in humans.

Future studies should evaluate the molecular characterization of distinct RE protocols in order to understand the biological response of skeletal muscle and optimize the development of training methods. Experimental models characterized by skeletal muscle wasting should also be investigated in order to propose RE as a nonpharmacological tool for humans. Additionally, this experimental model allows investigations regarding other stimulus associated with RE, that is, nutritional, pharmacological, and so forth.

\section{Conflict of Interests}

H. Nicastro, N. E. Zanchi, and A. H. Lancha Jr. have applied for a patent as inventors of University of São Paulo (USP) and FAPESP for the equipment developed (register no 018110024749).

\section{Acknowledgments}

H. Nicastro, N. E. Zanchi, and A. H. Lancha Jr. conceived and designed the project, drafted the paper and approved the final version of the paper; C. R. da Luz and D. F. S. Chaves drafted and approved the final version of the paper; C. R. da Luz designed the project. This project was supported by Fundação de Amparo a Pesquisa do Estado de São Paulo (FAPESP) through Grant 2008/51090-1; H. Nicastro, N. E. Zanchi, C. R. da Luz, and D. F. S. Chaves are supported by FAPESP (Grants 2010/07062-3; 2010/52561-8; 2011/046906; 2009/52022-2, resp.).

\section{References}

[1] J. A. Hawley, "Exercise as a therapeutic intervention for the prevention and treatment of insulin resistance," Dia-
betes/Metabolism Research and Reviews, vol. 20, no. 5, pp. 383393, 2004.

[2] V. D. S. Painelli, B. Gualano, G. G. Artioli et al., "The possible role of physical exercise on the treatment of idiopathic inflammatory myopathies," Autoimmunity Reviews, vol. 8, no. 5, pp. 355-359, 2009.

[3] G. Guilhem, C. Cornu, and A. Guével, "Neuromuscular and muscle-tendon system adaptations to isotonic and isokinetic eccentric exercise," Annals of Physical and Rehabilitation Medicine, vol. 53, no. 5, pp. 319-341, 2010.

[4] L. O. Pereira and A. H. Lancha Jr., "Effect of insulin and contraction up on glucose transport in skeletal muscle," Progress in Biophysics and Molecular Biology, vol. 84, no. 1, pp. 1-27, 2004.

[5] M. Izquierdo, K. Häkkinen, J. Ibáñez, W. J. Kraemer, and E. M. Gorostiaga, "Effects of combined resistance and cardiovascular training on strength, power, muscle cross-sectional area, and endurance markers in middle-aged men," European Journal of Applied Physiology, vol. 94, no. 1-2, pp. 70-75, 2005.

[6] J. J. Hulmi, S. Walker, J. P. Ahtiainen, K. Ny man, W. J. Kraemer, and K. Hakkinen, "Molecular signaling in muscle is affected by the specificit y of resistance exercise protocol," Scandinavian Journal of Medicine \& Science in Sports. In press.

[7] M. L. Leal, L. Lamas, M. S. Aoki et al., "Effect of different resistance-training regimens on the WNT-signaling pathway," European Journal of Applied Physiology, vol. 111, no. 10, pp. 2535-2545, 2011.

[8] L. Lamas, M. S. Aoki, C. Ugrinowitsch et al., "Expression of genes related to muscle plasticity after strength and power training regimens," Scandinavian Journal of Medicine and Science in Sports, vol. 20, no. 2, pp. 216-225, 2010.

[9] H. Roschel, C. Ugrinowistch, R. Barroso et al., "Effect of eccentric exercise velocity on akt/mtor/p70 s6k signaling in human skeletal muscle," Applied Physiology, Nutrition and Metabolism, vol. 36, no. 2, pp. 283-290, 2011.

[10] M. Neves Jr., G. Barreto, L. Boobis et al., "Incidence of adverse events associated with percutaneous muscular biopsy among healthy and diseased subjects," Scandinavian Journal of Medicine and Science in Sports. In press.

[11] M. A. Tarnopolsky, E. Pearce, K. Smith, and B. Lach, "Suctionmodified Bergström muscle biopsy technique: experience with 13,500 procedures," Muscle and Nerve, vol. 43, no. 5, pp. 717$725,2011$.

[12] H. Klitgaard, "A model for quantitative strength training of hindlimb muscles of the rat," Journal of Applied Physiology, vol. 64, no. 4, pp. 1740-1745, 1988.

[13] G. R. Adams, F. Haddad, P. W. Bodell, P. D. Tran, and K. M. Baldwin, "Combined isometric, concentric, and eccentric resistance exercise prevents unloading-induced muscle atrophy in rats," Journal of Applied Physiology, vol. 103, no. 5, pp. 1644-1654, 2007.

[14] F. Haddad, G. R. Adams, P. W. Bodell, and K. M. Baldwin, "Isometric resistance exercise fails to counteract skeletal muscle atrophy processes during the initial stages of unloading," Journal of Applied Physiology, vol. 100, no. 2, pp. 433-441, 2006.

[15] O. Wirth, E. W. Gregory, R. G. Cutlip, and G. R. Miller, "Control and quantitation of voluntary weight-lifting performance of rats," Journal of Applied Physiology, vol. 95, no. 1, pp. 402$412,2003$.

[16] J. D. Fluckey, E. E. Dupont-Versteegden, D. C. Montague et al., "A rat resistance exercise regimen attenuates losses of musculoskeletal mass during hindlimb suspension," Acta Physiologica Scandinavica, vol. 176, no. 4, pp. 293-300, 2002. 
[17] N. E. Zanchi, M. A. D. S. Filho, F. S. Lira et al., "Chronic resistance training decreases MuRF-1 and Atrogin-1 gene expression but does not modify Akt, GSK-3 $\beta$ and p70S6K levels in rats," European Journal of Applied Physiology, vol. 106, no. 3, pp. 415-423, 2009.

[18] H. Nicastro, N. E. Zanchi, C. R. da Luz et al., "Effects of leucine supplementation and resistance exercise on dexamethasoneinduced muscle atrophy and insulin resistance in rats," Nutrition. In press. 

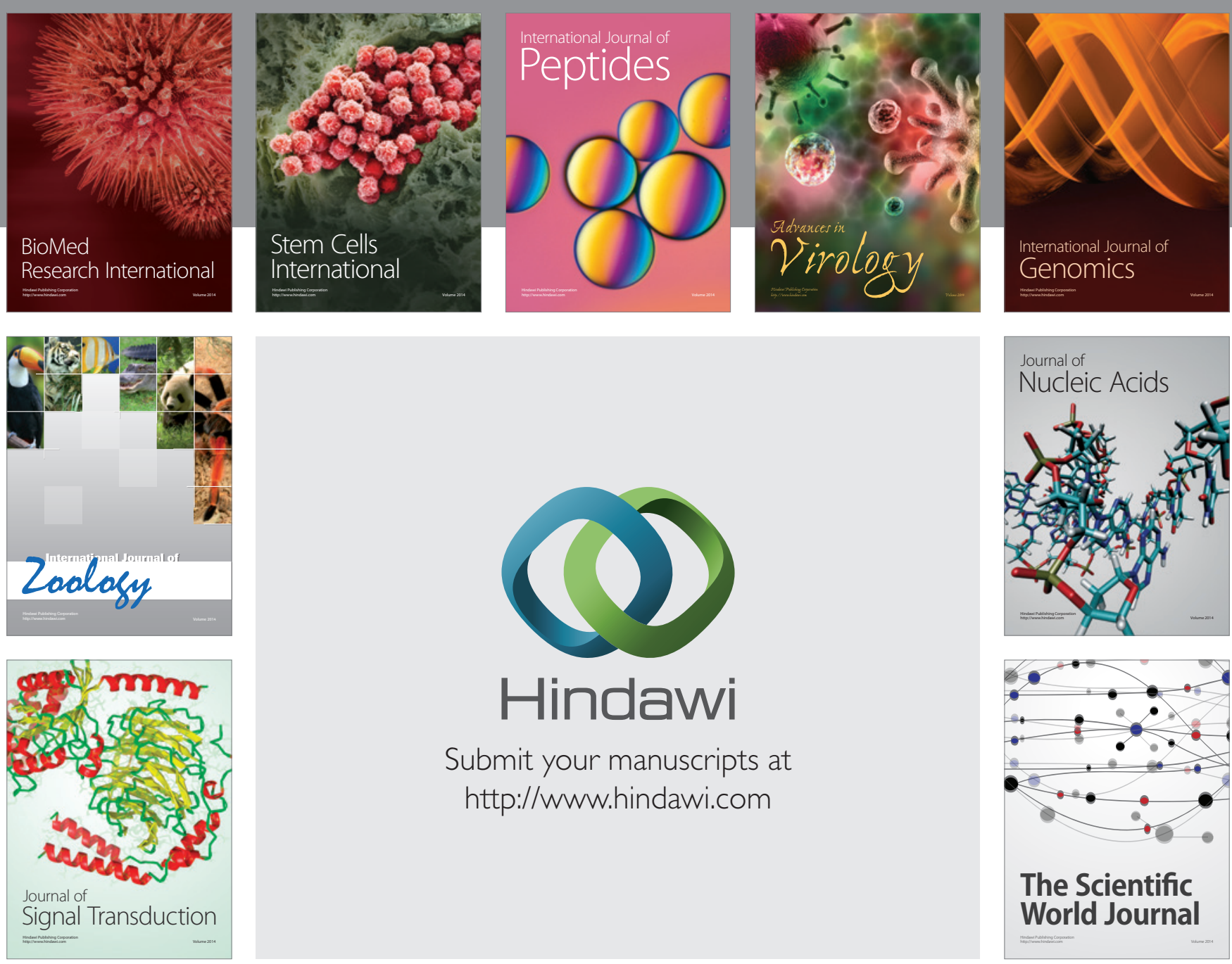

Submit your manuscripts at

http://www.hindawi.com
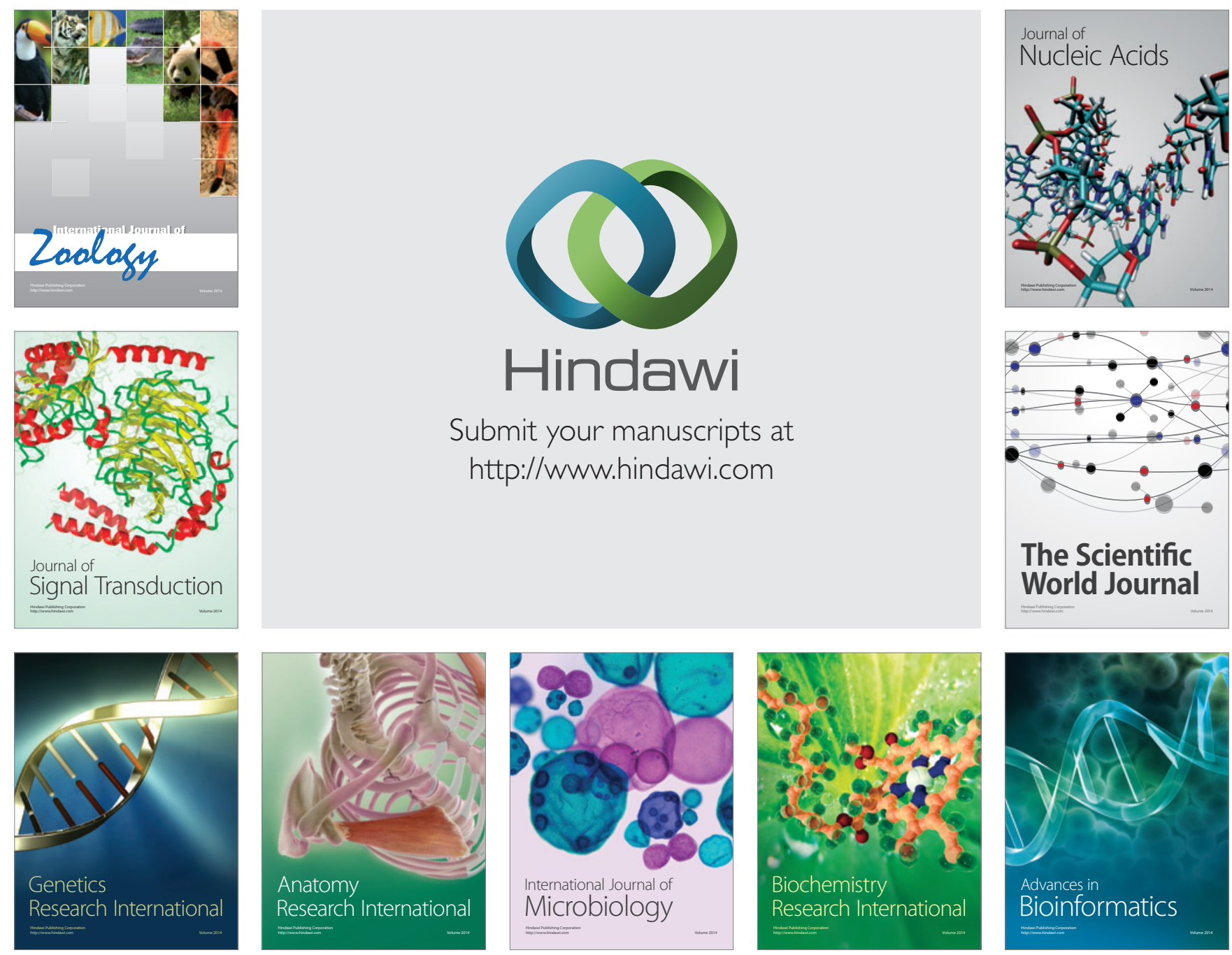

The Scientific World Journal
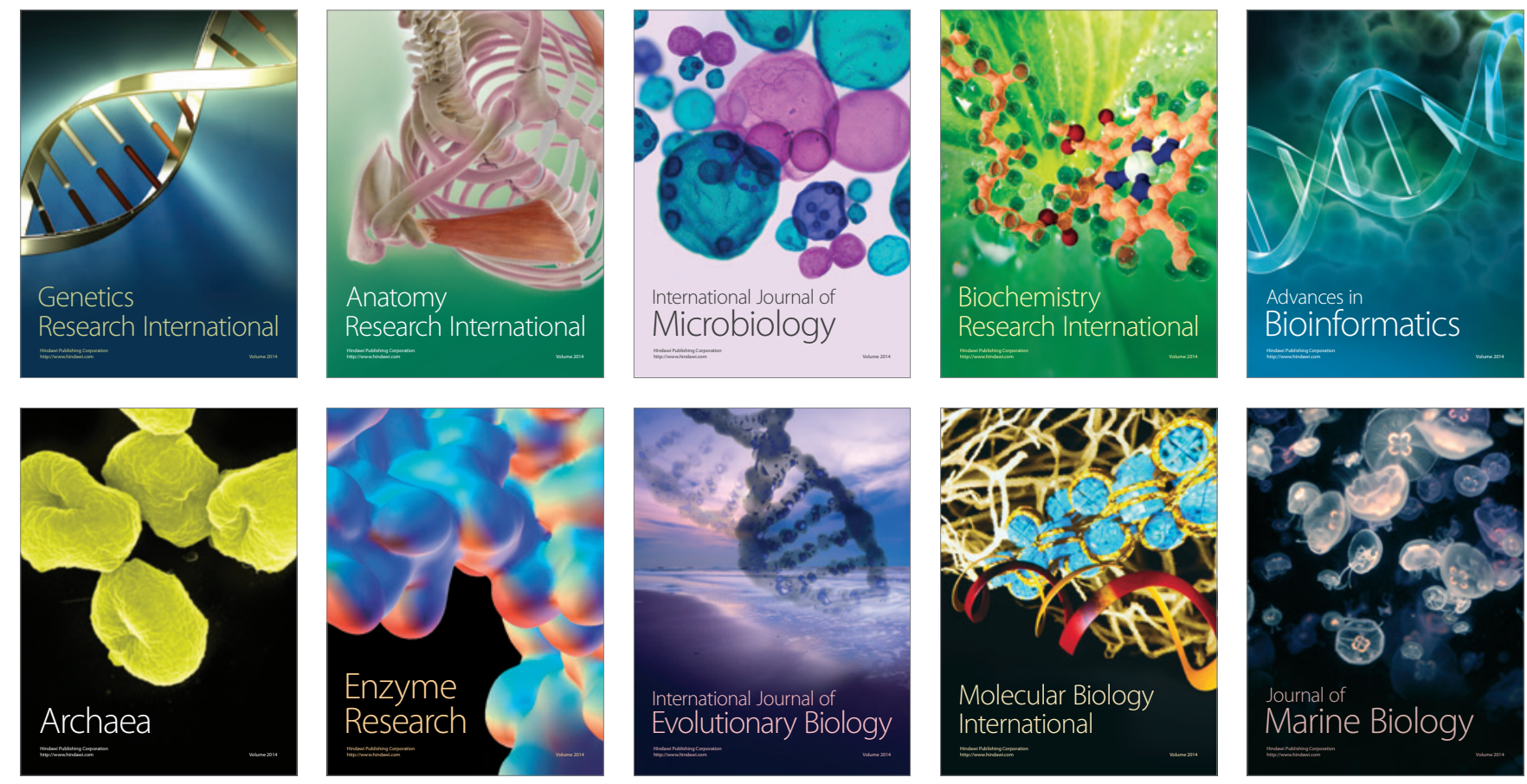